\title{
I-ACTSS-COVID-19—-the Italian acute care and trauma surgery survey for COVID-19 pandemic outbreak
}

\author{
Valerio Cozza ${ }^{1} \cdot$ Pietro Fransvea $^{1} \cdot$ Antonio La Greca $^{1}$ (D) Paolo De Paolis $^{2} \cdot$ Pierluigi Marini $^{3} \cdot$ Mauro Zago $^{4} \cdot$ \\ Gabriele Sganga ${ }^{1} \cdot$ I.-ACTSS.-COVID19 Collaborative Study Group
}

Received: 13 May 2020 / Accepted: 10 June 2020 / Published online: 24 June 2020

(C) Italian Society of Surgery (SIC) 2020

\begin{abstract}
The sudden COVID-19 outbreak in Italy has challenged our health systems and doctors faced the challenge of treating a large number of critically ill patients in a short time interval. Acute care surgeons, although not directly involved in treating COVID-19 + patients, have often modified their daily activity to help in this crisis. We have designed the first Italian survey on the effect of COVID-19 outbreak on Acute Care Surgery activity and submitted it to emergency surgeons in all the country to evaluate the experiences, trends, attitudes and possible educational outcomes that this emergency brought to light. A total of 532 valid surveys were collected during the study period. Lombardy and Lazio had the major answer rate. 96\% of responders noticed a decrease in surgical emergencies. The outbreak affected regions and hospitals in different ways depending on the local incidence of infection. Half of responders modified their approach to intra-abdominal infections towards a more conservative treatment. $43 \%$ of responders, mainly in the North, were shifted to assist non-surgical patients. There has been a direct but non-homogeneous involvement of emergency surgeons. Almost all hospitals have responded with specific pathways and training. Both emergency surgery and trauma activity have changed and generally decreased but the majority of surgeons have operated on suspected COVID-19 patients.
\end{abstract}

Keywords COVID-19 $\cdot$ Healthcare workers $\cdot$ Pandemic $\cdot$ Outbreak $\cdot$ Emergency $\cdot$ Acute care surgery

Abbreviations
$\begin{array}{ll}\text { COVID-19 } & \text { Coronavirus disease } 2019 \\ \text { PPE } & \text { Personal Protection Equipment } \\ \text { ACS } & \text { Acute Care Surgery }\end{array}$

Electronic supplementary material The online version of this article (https://doi.org/10.1007/s13304-020-00832-4) contains supplementary material, which is available to authorized users.

Antonio La Greca

antonio.lagreca@unicatt.it

1 Emergency Surgery and Trauma-Fondazione Policlinico Universitario “A. Gemelli” IRCCS, Università Cattolica del Sacro Cuore, Largo A. Gemelli 8, 00168 Rome, Italy

2 President of Società Italiana Di Chirurgia (SIC), Italian Society of Surgery, Rome, Italy

3 President of Associazione Chirurghi Ospedalieri Italiani (ACOI), Italian Association of Hospital Surgeons, Rome, Italy

4 President of Società Italiana Di Chirurgia D'Urgenza E Trauma (SICUT), Italan Society of Trauma and Emergency Surgery, Rome, Italy

\begin{tabular}{|c|c|}
\hline A.C.O.I & $\begin{array}{l}\text { Associazione Chirurghi Ospedalieri Ital- } \\
\text { ian-iItalian Association of Hospital } \\
\text { Surgeons }\end{array}$ \\
\hline S.I.C.U.T & $\begin{array}{l}\text { Società Italiana di Chirurgia d'Urgenza e } \\
\text { Trauma-Italian Society of Trauma and } \\
\text { Acute Care Surgery }\end{array}$ \\
\hline S.I.C & $\begin{array}{l}\text { (Società Italiana di Chirurgia-Italian Soci- } \\
\text { ety of Surgery }\end{array}$ \\
\hline RS & Respondent Surgeon \\
\hline Phd & Doctor of Philosophy \\
\hline ICU & Intensive Care Unit \\
\hline ED & Emergency Department \\
\hline
\end{tabular}

\section{Introduction}

The sudden COVID-19 outbreak in Italy has challenged our Hospitals to deal with an exceeding number of critically ill patients in a short time interval. This not only engaged multiple professionals in taking care of severe patients (even those not usually involved in critical care), but also compelled the entire hospital organizations to build new patient pathways 
(or even new hospitals), to organize different operators time shifts, to use new devices such as filters or personal protection equipment (PPE) and even to consider totally new safety issues for both patients and operators. All this represented a real and sudden mass casualty $[1,2]$.

Acute Care Surgery (ACS) practice might not be necessarily on the frontline of COVID-19+ patient care, but still ordinary emergencies must be dealt with and also acute surgical diseases may affect COVID-19 + patients, introducing the need for different clinical pathways and even new surgical approaches. Are we prepared for this challenge? In addition, how did the outbreak affect the activity of acute care surgery units?

In light of this, the aim of the present survey is to provide the first overview of the current Italian scenario concerning acute care and trauma surgery during the COVID-19 outbreak and to evaluate the experiences, trends, attitudes and possible educational outcomes that this emergency brought to light. The secondary endpoint is to identify any COVID19-related gross change in surgical emergencies workload in terms of number as well as type of cases.

\section{Methods}

A 56-item survey (see Supplementary material) was designed by two Italian Acute Care Surgeon Consultants (P.F. and V.C.) who work in the Acute Care and Trauma division at a University teaching hospital identified as a COVID-19+ regional referral centre (Hub/CTS). The survey was supervised and edited by the senior consultant (ALG) and the head of the department (GS). A second supervision and editing was performed by the scientific board of the A.C.O.I. (Associazione Chirurghi Ospedalieri Italiani - Italian Association of Hospital Surgeons), the scientific board of the S.I.C.U.T. (Società Italiana di Chirurgia d'Urgenza e Trauma-Italian Society of Trauma and Acute Care Surgery) and the scientific board of the S.I.C. (Società Italiana di Chirurgia-Italian Society of Surgery). The survey was designed in accordance with Checklist for Reporting Results of Internet E-Surveys (CHERRIES), Enhancing the QUAlity and Transparency Of health Research (SRQR Guidelines) and Consolidated criteria for reporting qualitative studies (COREQ): a 56-item checklist was uploaded on SurveyMonkey ${ }^{\circledR}$ and distributed via e-mail and social media (WhatsApp, Messenger) to Italian Emergency and Trauma Surgery physicians (herein after "consultants"), including both general surgeons rotating in the emergency duties as well as dedicated emergency and trauma surgeons, working both in COVID-19 as well as non-COVID-19 hospitals. Last year, general surgery trainees were also included. The responders were able to review and change the answers until the final submission of the questionnaire. No identifying or sensitive data were collected. Contact details were obtained from A.C.O.I., S.I.C.U.T. and S.I.C. email databases and from Universities and colleagues that agreed to support the project; the survey was distributed from 31 March 2020-2019 April 2020 (for a total of 20 days). The survey consisted of five sections:

- Section one: general information of the respondents (type of hospital, Italian region of origin, hospital role, hospital and ICU capacity, date of first COVID-19+ isolation, specific COVID-19 + pathway and ward);

- Section two: general and specific questions about Surgical activity (impact of COVID-19 + outbreak on surgery workload, changing in acute care surgery and trauma workload and organization, identification of a specific triage, theatre, ward, for both suspected and COVID19 + surgical patients, any change to the approach to most common surgical emergencies, any change to the laparoscopic approach, any specific online or on field training on COVID-19+;

- Section three: general and specific questions about ward duty (schedule modification, ward workload of COVID + patient in the surgical department, any changing in antibiotic treatment for COVID-19+ surgical patients);

- Section four: specific questions about impact on health professionals in the context of Acute Care Surgery (specific training on Personal Protection Equipment (PPE), any type of screening for acute care surgery healthcare workers);

- Section five: specific questions on research and training (trainee responsibilities, any modification in trainees rotation, expectation regarding any type of updating with guidelines/protocols on COVID-19 outbreak by surgical scientific societies);

The surveys with more than $10 \%$ of missed answers, with trivializing answers, and those not answered by colleagues involved in acute care surgery were excluded from the study. Data were entered into a Microsoft Excel (version 14.0) [Microsoft, Albuquerque New Mexico, USA] database and transferred to SofastatsTM for Windows [Paton-Simpson \& Associates Ltd,1.4.6/2 January 2016]. A descriptive 
statistical analysis was performed. Continuous and not normally distributed variables are presented as mean and or as a percentage $(\%)$.

\section{Results}

The survey reached about 1200 professionals involved in emergency and trauma care; a total of 532 valid answers were collected during the study period. This represents an estimated response rate of $44.3 \%$. All the respondents completed the survey (completion rate: $100 \%$ ).

The geographical origin of respondent surgeons (RS) is depicted in Fig. 1. Most of responders were from Lazio (22.20\%, $118 \mathrm{RS})$, Lombardy (17\%, $90 \mathrm{RS})$ and Tuscany (6.95\% 37 RS). A significant part of the RS, 342 (64.29\%), were consultants (as defined above), followed by 70 (13.16\%) Heads of Department and 60 (11.28\%) last year residents in training. Others RS such as fellows and $\mathrm{PhD}$ candidates are $11.28 \%$. Table 1 and Fig. 2 summarize hospital type and capacity: the most frequent were academic teaching hospitals (196 RS, 36.8\%), and 77.4\% (412 RS) were hospitals identified as COVID-19 centre. Moreover, 405 of the RS work in hospitals previously involved in trauma care: 212 RS (52.3\%) in a Hub centre (Centro Trauma Specialistico/CTS), 133 RS (32.8\%) in a Spoke centre (Centro Trauma di Zona/CTZ), and 60 RS (14.8\%) in a First Aid for trauma care (Pronto Soccorso Trauma/PST). The first COVID-19+ patient was most commonly isolated between March 1st-10th (52.6\%, 280 RS), while just a few hospitals isolated a COVID-19+ patient after March 20th (6.2\%, 33 RS). Tables 2 and 3 summarize the impact of COVID-19 outbreak on hospital set-up and on surgical activity, and Table 4 shows the impact of COVID-19 outbreaks on trauma care. Globally, most of the RS answered that their hospital had one or more wards specifically dedicated for COVID-19 + patients as well as dedicated ICU (348, $97.4 \%$ and $331,92.7 \%$, respectively), while suspected COVID$19+$ patients shared wards with positive patients in $53.5 \%$ of hospitals. 250 (70\%) of the RS answered that their hospitals directly analyse nasal/pharyngeal swabs even if in the $11.2 \%$ it is validated in a referral laboratory. Regarding surgical activity, more than half of the RS hospitals $(245,57.2 \%)$ have preserved

Table 1 Hospital type and capacity

\begin{tabular}{ll}
\hline Definition of the Hospital & $N(\%)$ \\
Academic Teaching Hospital & $196(36.8)$ \\
Regional Hospital & $124(23.3)$ \\
Urban Hospital & $171(32.1)$ \\
Private Hospital & $33(6.20)$ \\
Other & $8(1.50)$ \\
Previously involved in trauma care & $N(\%)$ \\
Hub centre (CTS) & $212(52.3 \%)$ \\
Spoke centre (CTZ) & $133(32.8 \%)$ \\
First Aid for trauma care (PST) & $60(14.8 \%)$ \\
Hospital capacity & $N(\%)$ \\
$\leq 500$ beds & $258(48.5)$ \\
$>500$ beds & $274(51.5)$ \\
Standard ICU capacity & $N(\%)$ \\
$\leq 20$ beds & $286(53.7)$ \\
$>20$ beds & $246(46.2)$ \\
\hline
\end{tabular}

Fig. 1 Geographical origin of respondent surgeons

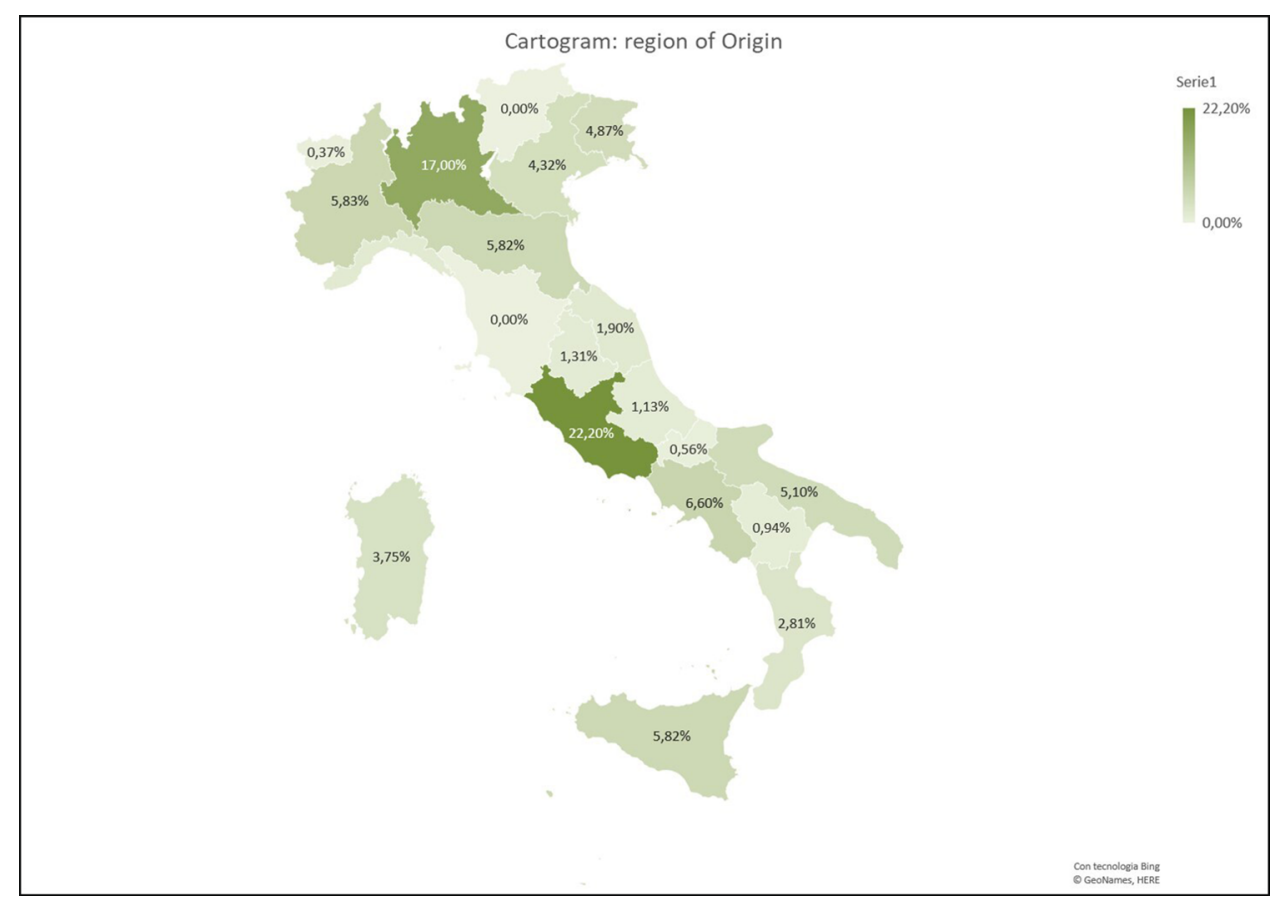




\section{Working in a COVID19 Hospital}

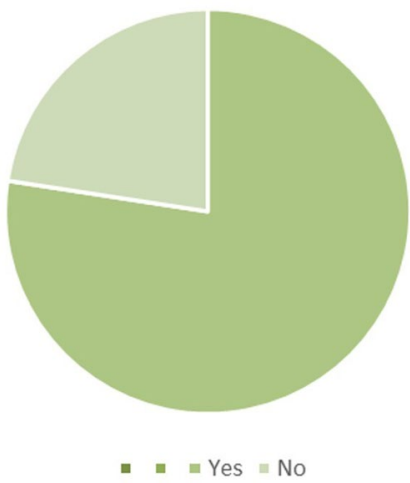

only non-postponable cancer surgery, 97 RS (22.6\%) cancelled all elective surgery, while just 13 RS (3.0\%) maintained normal surgical practice for both elective and emergency. Numbers of surgical procedures performed on suspected COVID-19 or on positive patients are highlighted in Fig. 3. Most of the RS (299 RS, 73.6\%) have operated on less than five COVID19 + patients and only $17.14 \%$ have performed surgical procedures on more than 11 COVID-19+ patients. Focusing on surgical activity this survey showed how half of the RS $(53.2 \%, 201 \mathrm{RS})$ have or are planning to have a specific surgical pathway (PPE, specific triage) for the COVID-19+ surgical patients, and in most of the cases the pre-operative pathway includes a chest CT for every patient $(68.5 \%, 278 \mathrm{RS})$. In addition, 271 RS (63.3\%) have identified a dedicated COVID-19

Fig. 2 Hospital type and capacity

Table 2 Impact of COVID-19 outbreak on hospital set-up

\begin{tabular}{|c|c|c|c|c|c|c|}
\hline & & \multicolumn{2}{|l|}{ Yes, $n .(\%)$} & \multicolumn{3}{|l|}{ No, $n .(\%)$} \\
\hline \multicolumn{2}{|c|}{ Dedicated wards for COVID -19 positive patients? } & \multicolumn{2}{|l|}{$348(97.4)$} & \multicolumn{3}{|l|}{$9(2.5)$} \\
\hline \multicolumn{2}{|c|}{ Specific ICU for COVID -19 positive patients? } & \multicolumn{2}{|l|}{$331(92.7)$} & \multicolumn{3}{|l|}{$24(6.7)$} \\
\hline \multicolumn{2}{|c|}{ Increase the number of ICU beds after the outbreak? } & \multicolumn{2}{|l|}{$329(92.2)$} & \multicolumn{3}{|l|}{$28(7.8)$} \\
\hline \multirow{2}{*}{\multicolumn{2}{|c|}{ Suspected COVID-19 and COVID-19 share wards }} & \multicolumn{2}{|c|}{$191(53.5)$} & \multicolumn{3}{|l|}{$166(46.5)$} \\
\hline & & \multicolumn{2}{|c|}{$\begin{array}{l}\text { To a COVID-19 ward with mixed } \\
\text { surgical and medical patients }\end{array}$} & \multicolumn{2}{|c|}{$\begin{array}{l}\text { To a COVID- } 19 \text { area inside, } \\
\text { a normal surgical ward }\end{array}$} & $\begin{array}{l}\text { To a specific surgi- } \\
\text { cal COVID-19 } \\
\text { ward }\end{array}$ \\
\hline \multicolumn{2}{|c|}{$\begin{array}{l}\text { Admission of suspect COVID-19 or COVID- } 19+\text { surgi- } \\
\text { cal patient }\end{array}$} & $177(49.5 \%)$ & & $58(16.2 \%)$ & & $122(34.2)$ \\
\hline & \multicolumn{2}{|c|}{$\begin{array}{l}\text { To a COVID- } 19 \text { ward with mixed } \\
\text { surgical and medical patients }\end{array}$} & \multicolumn{2}{|c|}{$\begin{array}{l}\text { To a COVID- } 19 \text { area inside, a } \\
\text { normal surgical ward }\end{array}$} & \multicolumn{2}{|c|}{$\begin{array}{l}\text { To a specific surgical COVID-19 } \\
\text { ward }\end{array}$} \\
\hline $\begin{array}{l}\text { Admission of suspect COVID-19 } \\
\text { or COVID-19+ surgical patient }\end{array}$ & \multicolumn{2}{|l|}{$177(49.5 \%)$} & \multicolumn{2}{|l|}{$58(16.2 \%)$} & \multicolumn{2}{|l|}{$122(34.2)$} \\
\hline
\end{tabular}

Table 3 Impact of COVID-19 outbreak on surgical activity

\begin{tabular}{|c|c|c|c|c|c|c|}
\hline & $\begin{array}{l}\text { Maintaining both elec- } \\
\text { tive and emergency } \\
\text { surgery }\end{array}$ & $\begin{array}{l}\text { Only non-post- } \\
\text { ponable cancer } \\
\text { surgery }\end{array}$ & $\begin{array}{l}\text { Cancelling } \\
\text { all elective } \\
\text { surgery }\end{array}$ & Complete lockdown & $\begin{array}{l}\text { Elective surgery } \\
\text { addressed to other des- } \\
\text { ignated hospitals }\end{array}$ & Other \\
\hline \multirow{3}{*}{$\begin{array}{l}\text { General impact of } \\
\text { COVID-19 outbreak } \\
\text { on surgical activity }\end{array}$} & $13(3 \%)$ & $245(57.2 \%)$ & $97(22.7 \%)$ & $11(2.6 \%)$ & $3.3 \%$ & $1.9 \%$ \\
\hline & & & & & 14 & 8 \\
\hline & $90-100 \%$ & $50-89 \%$ & $25-49 \%$ & $<25$ & & \\
\hline \multirow{2}{*}{$\begin{array}{l}\text { Current rate of emer- } \\
\text { gency surgical activity } \\
\text { as compared to } \\
\text { standard }\end{array}$} & $33(7.7 \%)$ & $79(18.5 \%)$ & $131(30.6 \%)$ & $185(43.2 \%)$ & & \\
\hline & Yes, $n .(\%)$ & No, $n .(\%)$ & & & & \\
\hline \multirow{2}{*}{$\begin{array}{l}\text { Any surgery performed } \\
\text { on a COVID-19 } \\
\text { patient }\end{array}$} & $256(59.8)$ & $172(40.2)$ & & & & \\
\hline & Yes, $n .(\%)$ & No, $n .(\%)$ & & & & \\
\hline $\begin{array}{l}\text { Dedicated COVID-19 } \\
\text { operating theatre }\end{array}$ & $271(63.3)$ & $157(36.9)$ & & & & \\
\hline
\end{tabular}


Table 4 Impact of COVID-19 outbreaks on Trauma care

\begin{tabular}{|c|c|c|c|c|}
\hline & Trauma hub & $\begin{array}{l}\text { Trauma hub but other hospitals } \\
\text { receive the majority of trauma } \\
\text { patients }\end{array}$ & $\begin{array}{l}\text { We transfer trauma as soon as pos- } \\
\text { sible to other dedicated hospitals }\end{array}$ & $\begin{array}{l}\text { Not a trauma } \\
\text { hub anymore }\end{array}$ \\
\hline \multirow[t]{2}{*}{ Trauma care set-up } & $184(39.8 \%)$ & $60(12.9 \%)$ & $64(13.8 \%)$ & $154(33.3 \%)$ \\
\hline & Yes, $n .(\%)$ & No, $n .(\%)$ & & \\
\hline \multirow[t]{2}{*}{ Trauma care workload changed } & $293(68.4)$ & $135(31.5)$ & & \\
\hline & $90-100 \%$ & $50-89 \%$ & $25-49 \%$ & $<25 \%$ \\
\hline $\begin{array}{l}\text { Current trauma care workload as } \\
\text { compared to before }\end{array}$ & $17(4.7 \%)$ & $38(10.5 \%)$ & 105 (28.9) & $203(55.9 \%)$ \\
\hline
\end{tabular}

operating theatre even if in just one-third of cases with negative pressure ventilation system (83 RS, 32.4\%).

Moreover, half of the RS $(221,54,4 \%)$ have performed or would consider performing laparoscopies on COVID$19+$ patients, all of them adopting specific measures such us homemade filters for gas evacuation, aspiration devices, negative operative theatre pressure. Investigating the approach to the most common diseases faced in an acute care setting such as acute appendicitis, acute cholecystitis and acute diverticulitis, this survey shows a global increase in conservative treatment for all of these (Fig. 4). Moreover, in case of fever and suspected intra-abdominal infection, most of patients follow a specific COVID-19 pathway both in medicine and surgical wards $(47.1 \%, 178 \mathrm{RS}$, and $26.9 \%$, $102 \mathrm{RS}$, respectively). However, the majority of the RS $(94.2 \%, 326 \mathrm{RS})$ follow the same peri-operative antibiotic protocol as for non-COVID-19+.

Regarding COVID-19+ patient undergoing surgical procedures, only 21 RS (5.6\%) encountered a higher postoperative complications rate while the majority of the RS (71.7\%, 271 RS) had not enough patients to give an answer. In addition, $107 \mathrm{RS}$ answered that they have encountered COVID-19+ patients, already hospitalized, who developed abdominal condition. Table 5 and Fig. 5 show the impact of COVID-19 outbreak on healthcare workers. Almost half of the RS $(149,43.8 \%)$ were shifted to assist not surgical COVID-19+ patients. Most of the RS (66.1\%, $250 \mathrm{RS})$ say that they have never been involved in any simulation for mass casualties. However, after the COVID-19 outbreak, $45.2 \%$ have already participated to some kind of COVID19 dedicated training $(22.49 \%$ on line training, $22.75 \%$ on field training) and $159 \mathrm{RS}(46.7 \%)$ had a specific training on PPE. Moreover, 59.03\% (219 RS) have modified their time schedule, by setting-up a dedicated staff for COVID-19, or by minimising the number of staff on duty. Table 6 shows the impact of COVID-19 outbreak on residents in training.

The answer to the last question was the same for almost all of the RS: the $97.0 \%$ expect updated guidelines/protocols on COVID-19 outbreak from surgical scientific societies.

\section{Discussion}

The COVID-19 outbreak has significantly affected healthcare systems across the world. Priorities of care have been reconsidered and hospitals are suffering mainly with an
Fig. 3 Numbers of surgical procedures performed on suspected COVID-19 or on positive patients

\section{Emergecy surgery procedure}

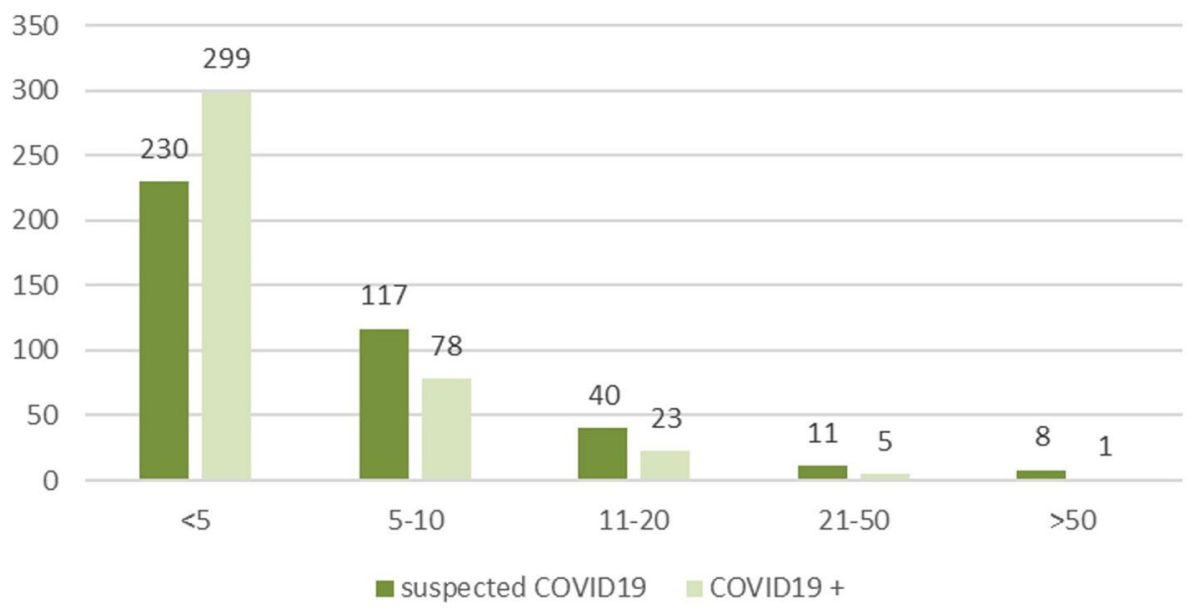


Fig. 4 Approach to the most common diseases

\section{Approach}

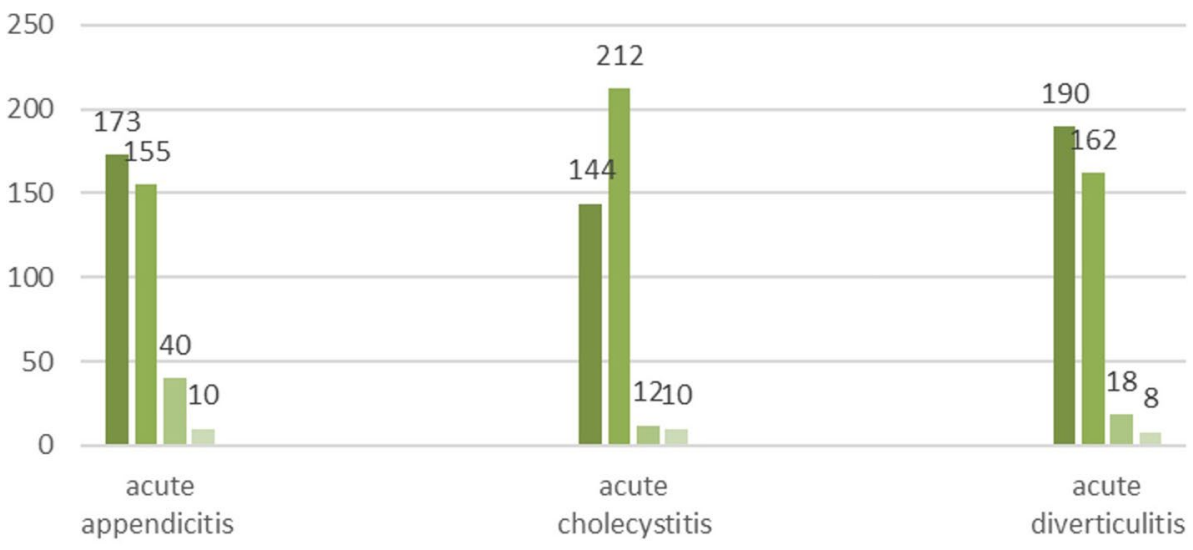

Not at all $\square$ increased conservative treatment $\square$ increased open procedures $\square$ Other

Table 5 Impact of COVID-19 outbreak on healthcare workers

\begin{tabular}{|c|c|c|c|}
\hline & Without swab & With swab & No \\
\hline Acute care surgery healthcare screened for COVID-19, even without symptoms & $40(11.8 \%)$ & $44(12.9 \%)$ & $256(75.3 \%)$ \\
\hline Acute care surgery healthcare workers get screened for COVID-19 when they develop symptoms & $45(13.2 \%)$ & $253(74.4 \%)$ & $42(12.3 \%)$ \\
\hline Acute care surgery healthcare workers get screened for COVID-19 once exposed to a positive patient & $51(15 \%)$ & $161(47.3 \%)$ & $128(37.6 \%)$ \\
\hline
\end{tabular}

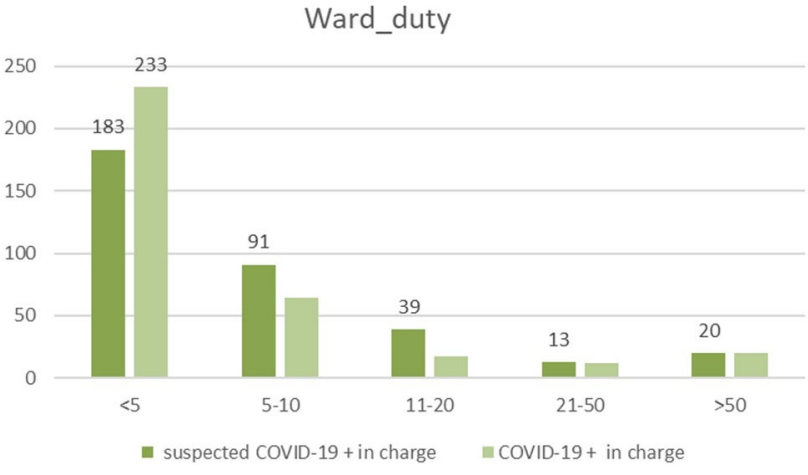

Fig. 5 Impact of COVID-19 outbreak on healthcare workers

unexpected load of patients requiring an enormous amount of resources (sometimes not available) [3]. At the time of this article, the country is entering the so-called "Phase II", corresponding to the flattening of the curve of cases and a partial and cautious release of the previous complete lockdown of social/economic activities. The world has diagnosed more than $3,500,000$ cases and almost 250,000 deaths; in Italy, we have reached 200,000 cases and more than 28,000 deaths. Lombardy, the most affected region, accounted for 77,000 cases and over 14,000 deaths. The flexibility of our health system has permitted to cope with these difficulties but routine activity has changed and
Table 6 Impact of COVID-19 outbreak and resident in training

\begin{tabular}{llll}
\hline & Yes, $n .(\%)$ & No $n .(\%)$ & \\
\hline Trainees in your team? & $187(55)$ & $153(45)$ & \\
$\begin{array}{c}\text { Changing trainee responsi- } \\
\text { bilities? }\end{array}$ & $83(44.6 \%)$ & $103(55.4 \%)$ & \\
$\begin{array}{c}\text { Do trainees assist COVID- } \\
19+\text { surgical patients? }\end{array}$ & $87(46.7 \%$ & $98(53.2)$ & \\
& Unchanged & Decreased & Increased \\
Trainee workload & $52(27.9 \%)$ & $115(61.8 \%)$ & $19(10.2 \%)$ \\
\hline
\end{tabular}

surgery has often been reduced to "real emergencies and cancers only". Sometimes surgeons have been redistributed to take care of the hospital emergency room, such as emergency doctors and/or to assist COVID + or internal medicine patients.

We have investigated the experience of emergency surgeons in our country, Italy, which has been among the first and the most affected ones. We have observed a substantial and rapid response to our questionnaire from the most involved region, Lombardy, in the first $48 \mathrm{~h}$. A solid participation came from the whole North of Italy: this confirms that regions more involved by a change of practice feel the importance of sharing and testifying their activity in order to achieve better outcomes. 
Almost all participants indicated that their hospital identified a COVID-19 pathway, had dedicated Intensive Care Unit (ICU) for those patients and increased ICU beds. Likely, the caseload was a crucial factor, together with resources availability, for choosing to admit suspected and confirmed COVID-19 patients to specific surgical wards or in mixed medical and surgical COVID + wards.

Almost $2 / 3$ of hospitals had a surgical procedure for suspected or confirmed COVID19 + patients, and slightly less had a specific COVID-19 theatre. Interestingly enough, 89 (34.8\% of participants) do not know and are not concerned with the ventilation of the operating room, while the ventilation system is considered important for the stays of COVID + patients in intensive care.

Despite a significant decrease in their global load, general surgery emergencies in Italy still represent about $15 \%$ of Emergency Department (ED) admissions. Moreover, surgeons have changed their activity in response to the crisis: ward reduction/beds in mixed units, sometimes reallocation of surgeons, need to operate with PPE in dedicated theatres, change in residents' activity, and increase in conservative treatments for most common diseases [4]. More specifically, this survey showed that treatment options for both acute appendicitis and acute cholecystitis changed towards a conservative management, while there has been a slight increase of surgical procedures for complicated diverticulitis. This last finding may be due to the fact that patients affected by colonic diverticulosis are used to experience chronic recurrent abdominal pain and, frightened by the current epidemic, delay their arrival to the emergency department only after reaching more severe conditions.

An intensive debate is ongoing on indications, timing and procedures for surgical operations in suspected or confirmed COVID 19 patients [5, 6]. The main issues of the debate, as stated by descriptive comments of the respondents, are as follows: should we actually modify (increase) our indications to conservative treatments as for COVID-19 alone? Should we still consider laparoscopy for these patients? Which precautions should be considered mandatory in open and laparoscopy surgery? However, it is still early to assess the effect of these changes on outcomes, and future studies are needed.

Trauma care has decreased almost everywhere and it has been redistributed in one-third of cases to other hospitals as well.

Intra-abdominal infections, often admitted in a "COVID19 pathway" for similarity of symptoms, have been treated more frequently by physicians instead of surgeons. The longterm outcomes of this shift will be probably investigated in the next months.

The activity of trainees has changed in different ways: some of them were still treating acute/surgical patients (with the same or reduced volume), some were shifted to assist
COVID patients. Another group has reduced or stopped clinical activity. Some others some of them have even been moved from a residency position to a contract of employment some months ahead of their formal degree to cope with shortage of staff. We believe this depends on a longstanding issue due to the double role of Italian trainees (students and healthcare providers) but mainly on the impact of the pandemic on the different regions and hospitals.

Surgery has been rarely performed on a large number of COVID-19 positive patients, but almost half of the surgeons performed operation on suspected cases. The long-term outcome of these operations still need to be investigated.

This survey shows a strong interest on the part of acute care surgeons, involved as "first line" operators in managing the pathways of suspected and confirmed patients and often directly treating COVID-19 patients. Since emergencies are not among delayable/deletable operations, one could expect issues in terms of non-available ICU beds or theatres. The reduction of total number of cases (people staying home longer than before), redistribution of non COVID-19 emergencies to COVID-free hospitals and reduction of elective cases probably allowed not to reach a critical level. Overturning the medal, the outcome of surgical emergencies (mainly delayed diagnosis due to "COVID-19" pathway for fever and delayed treatment for late ED presentation) still needs to be further investigated $[7,8]$.

This study has some limitations.

The survey is addressed to individual operators in an anonymous form to involve and collect the largest possible number of health professionals and to get genuine and authentic answers. The regional distribution of respondents and their affiliation according to the trauma and non-trauma emergency network depict a reliable image of current perceptions of the outbreak. As a counterpart, the structure of the study does not allow to retrieve the hospitals where the respondents work in. In some regions, the hospital emergency network has been re-organized by identifying hubs (CTS) for severe trauma and emergency general surgery and hubs (CTS) for not postponable cancer and/or other type of surgery. Other regions with less impact of the pandemic have adopted a more flexible healthcare system. Some hospitals have been dedicated to COVID-19 only, again with different distribution by regions. The load of different types of surgery in different hospitals might have been a function of the regional re-distribution of surgical diseases and trauma care during the COVID-19 outbreak, accounting for a regional heterogeneity that this survey cannot accurately estimate.

The questions of the survey were not built to explore how a positive case was defined by each respondent. We know that different centres have activated different diagnostic pathways. Our Government Institutions, the Civil Protection Department over all, considered only swab positive patients as COVID-19 cases. This has been a matter of discussion 
during the past 2 months press conferences and within the scientific community and still represents an open epidemiological problem. The practical need to better predict which patients should be considered "positive" taking into account the false negative rate of the nasopharyngeal swabs and the urge to address patients to the right "clean" or "dirty" clinical pathway as early as possible (even before the technical time laps needed to obtain a swab result) still lacks a definitive standardized answer. We feel that, overall, this heterogeneity is an unavoidable part of the figures depicted by the survey, but it introduces a potential bias in the accurate estimate of COVID-19 patients workload and treatment choices and results.

\section{Conclusion}

COVID-19 outbreak has affected healthcare systems across the world, with an enormous amount of resources involved and predictable future effects on health, economics, social life and culture. Many studies have investigated the effects on our social environment in terms of psychology, education, work and economics. This is the first survey published on the emergency surgery setting. We have shown a direct but non-homogeneous involvement of emergency surgeons, mainly related to regional situations. Almost all hospitals have responded with specific pathways and training. PPEs use and screening are variable among hospitals. Both emergency surgery and trauma activity have changed and generally decreased but the majority of surgeons have operated on suspected COVID-19 patients. Intra-abdominal infections treatment has been shifted to a higher rate of nonoperative treatment and often transferred under the care of physicians due to common symptoms (fever, diarrhoea etc.). The outcome of these changes could be considered as "collateral damage" of COVID-19 infection and needs further investigation.

Acknowledgements The authors acknowledge all the brave Healthcare Professionals involved in the management of the epidemics outbreaks at Fondazione Policlinico Universitario A. Gemelli IRCCS, in Italy and all over the world. In particular doctors: Marta Di Grezia, Valeria Fico, Flavio Tirelli, Gilda Pepe, Maurizio Foco, Daniele Gui, Sabina Magalini, Mauro Pittiruti, Vincenzo La Vergata and all the nurses and residents in training actively involved in this pandemic outbreak.

Author contributions VC, PF Study conception and design, literature search, acquisition, interpretation and analysis of data, drafting and critically revising the article for important intellectual content; and final approval of the version to be published. ALG: drafting and critically revising the article for important intellectual content and final approval of the version to be published. PdP, PLM, MZ: acquisition of data and critically revising the article for important intellectual content. GS: drafting and critically revising the article for important intellectual content and final approval of the version to be published.

Data availability "Not applicable". Our manuscript does not contain data from any individual person, so this section is not applicable to our submission.

\section{Compliance with ethical standards}

Conflict of interest The authors declare no potential conflict/competing of interest related to this manuscript.

Research involving human participants and/or animals Our manuscript does not report on, or involve the use of any animal or human data or tissue, and does not contain data from any individual person, so this section is not applicable to our submission.

Informed consent Not applicable.

\section{References}

1. Livingston E, Bucher K (2020) Coronavirus Disease 2019 (COVID-19) in Italy. JAMA. https://doi.org/10.1001/ jama.2020.4344

2. Tong A (2007) Consolidated criteria for reporting qualitative research (COREQ): a 32-item checklist for interviews and focus groups. Int J Qual Health Care 19(6):349-357

3. Sohrabi C, Alsafi Z, O'Neill N et al (2020) World Health Organization declares global emergency: a review of the 2019 novel coronavirus (COVID-19). Int J Surg 76:71-76

4. Coccolini F, Sartelli M, Kluger Y et al (2020) COVID-19 the showdown for mass casualty preparedness and management: the Cassandra Syndrome. World J Emerg Surg 15(1):26

5. COVIDSurg Collaborative (2020) Global guidance for surgical care during the COVID-19 pandemic. Br J Surg. https://doi. org/10.1002/bjs.11646

6. Coimbra R, Edwards S, Kurihara H et al (2020) European society of trauma and emergency surgery (ESTES) recommendations for trauma and emergency surgery preparation during times of COVID-19 infection. Eur J Trauma Emerg Surg 46(3):505-510

7. Alhazzani W, Møller MH, Arabi YM et al (2020) Surviving sepsis campaign: guidelines on the management of critically ill adults with Coronavirus Disease 2019 (COVID-19). Intensive Care Med 46(5):854-887

8. Angelos G, Dockter AG, Gachabayov M, Latifi R, Bergamaschi R (2020) Emergency colorectal surgery in a COVID-19 pandemic epicenter. Surg Technol Int 36:18-21

Publisher's Note Springer Nature remains neutral with regard to jurisdictional claims in published maps and institutional affiliations. 